\title{
Nonlinear and multiscale dynamics of smart materials in energy harvesting
}

\author{
G. Litak ${ }^{1}$, E. Manoach ${ }^{2}$, and E. Halvorsen ${ }^{3}$ \\ 1 Faculty of Mechanical Engineering, Lublin University of Technology, 20-618 Lublin, \\ Poland \\ 2 Institute of Mechanics, Bulgarian Academy of Sciences, Acad. G. Bonchev Street, Bl. 4, \\ 1113 Sofia, Bulgaria \\ 3 Department of Micro- and Nanosystem Technology, Buskerud and Vestfold University \\ College, Campus Vestfold, Raveien 205, 3184 Borre, Norway
}

Received 9 October 2015 / Received in final form 9 October 2015

Published online 20 November 2015

This special issue is a result of discussions performed during a workshop (with the same name) held in Lublin, February 2014. This meeting served as the seed to invite several experts in the field to present contributions for this Special Topics issue which reflect the present state of the art for research and development of smart materials and their possible applications for energy control and energy harvesting.

The emerging technologies of battery-free wireless sensor networks, safetymonitoring devices and self-powered low-power electronics have stimulated studies on energy harvesting to convert mechanical vibration into electrical energy. Such a conversion can be used to harvest vibration energy and makes an autonomous operation possible providing environmental benefits through reduced use, and therefore reduced disposal, of batteries while giving lower maintenance costs and enabling sensors or small devices to work under difficult conditions or in hard-to-reach places. In most cases, ambient vibration energy is collected [1,2] as opposed to deliberately generated vibrations made for the sake of power transfer. As the type of ambient vibration can vary over time and may include components of low frequencies, possibly distributed over a significant relative bandwidth, it can be ineffective to use linear devices with high quality factor. These devices have their strongest merit when they work at their resonant frequency. Linear configurations with associated piezoelectric, electrostatic or electromagnetic transducers can be optimized by electrical impedance matching or advanced switching electronics [3]. Unfortunately, the frequency range for a linear system tend to be narrow. In the previous special issue we made a review of the smart materials couplings to mechanical systems and disscused possible applications of nonlinear composite materials and structures in engineering and energy harvesting [4].

In the meantime there were many attempts to broaden the bandwidth of the oscillations exhibited by a single-mechanical-degree-of-freedom harvester when it is excited. These attempts include designs involving multistable systems with a nonlinear effective stiffness which can be obtained by several means that are not necessarily purely mechanical. Both softening- and hardening spring characteristics of the 
nonlinear resonator have been explored. Both characteristics lead to multiple solutions consisting of more energetic resonant and less energetic nonresonant responses to the excitation [5-9]. Another way to get multiple frequencies was based on extending the degrees of freedom in the harvester resonator. Finally, in some special systems a hybrid energy harvesting concept could be more effective. In most cases the hybrid harvester is based on electromagnetic and piezoelectric transducers.

The application of smart and composite materials and structures and their internal nonlinear effects are very important to ensure a broad band frequency effect in the area of energy transduction. Consequently, the appearance of nonlinear resonance or multiscale solutions, typical to the nonlinear systems, needs special system identification procedures which are discussed in the present issue.

Based on the papers appearing in this issue we would like to distinguish several important categories with respect to the content, including: the dynamics of devices with piezoelectric and electromagnetic transducers (part $1 \& 2$, respectively), dynamics of the hybrid system (part 3), prospective materials and structures for energy harvesting (part 4), and finally, nonlinear identification, dynamics and control with smart materials (part 5). Note that the largest number of the examined systems are based on piezoelectric transducers [10-22]. While in conventional, macroscale engineering, electrical generators based on electromagnetic transduction dominate, in small-scale energy harvesting a piezoelectric energy conversion technique is competitive with electromagnetic ones [23-26]. A hybrid system [27] composed of two complementary concepts: electromagnetic and piezoelectric can be also used in a nonlinear system. This design ideally applies to the different solutions present in the nonlinear system where various receipts for energy harvesting fits better. It is also worth to notice that novel smart materials and structures which could be coupled to the mechanical resonator are continuously sought [28-31]. In this context new nonlinear control methods are developed to optimize the system performance and diagnose $[32,33]$.

We are very grateful for the support received from Lublin University of Technology and Polish National Science Center under the Grant No. 2012/05/B/ST8/00080 to sponsor this workshop.

\section{References}

1. P.D. Mitcheson, E.M. Yeatman, G.K. Rao, A.S. Holmes, T.C. Green, Proc. IEEE 96, 1457 (2008)

2. A. Erturk, J. Hoffmann, D.J. Inman, App. Phys. Lett. 94, 254102 (2009)

3. E. Lefeuvre, A. Badel, C. Richard, D. Guyomar, J. Intell. Mater. Syst. Struct. 16, 865 (2005)

4. G. Litak, E. Manoach, Eur. Phys. J. Special Topics 222, 1479 (2013)

5. R.L. Harne, K.W. Wang, Smart Mat. Struct. 22, 023001 (2013)

6. S.P. Pellegrini, N. Tolou, M. Schenk, J.L. Herder, J. Intell. Mater. Syst. Struct. 24, 1303 (2013)

7. J. Twiefel, H. Westermann, J. Intell. Mater. Syst. Struct. 24, 1291 (2013)

8. M.F. Daqaq, R. Masana, A. Erturk, D.D. Quinn, Appl. Mech. Rev. 66, 040801 (2014)

9. Y. Wang, L. Peng, Z. Huang, Theor. Appl. Mech. Lett. 5, 151 (2015)

10. Y. Bai, Z. Havranek, P. Tofel, C. Meggs, H. Hughes, T.W. Button, Eur. Phys. J. Special Topics 224(14-15), 2675 (2015)

11. Y. Zhang, R. Zheng, T. Kaizuka, D. Su, K. Nakano, M.P. Cartmell, Eur. Phys. J. Special Topics 224(14-15), 2687 (2015)

12. H. Madinei, H. Haddad Khodaparast, S. Adhikari, M.I. Friswell, M. Fazeli, Eur. Phys. J. Special Topics 224(14-15), 2703 (2015) 
13. A. Koszewnik, P. Grześ, W. Walendziuk, Eur. Phys. J. Special Topics 224(14-15), 2719 (2015)

14. H. Abdelmoula, A. Abdelkefi, Eur. Phys. J. Special Topics 224(14-15), 2733 (2015)

15. S. Stoykov, E. Manoach, G. Litak, Eur. Phys. J. Special Topics 224(14-15), 2755 (2015)

16. M. Borowiec, Eur. Phys. J. Special Topics 224(14-15), 2771 (2015)

17. W.O.V. Barbosa, A.S. De Paula, M.A. Savi, D.J. Inman, Eur. Phys. J. Special Topics 224(14-15), 2787 (2015)

18. A. Kumar, S.F. Ali, A. Arockiarajan, Eur. Phys. J. Special Topics 224(14-15), 2803 (2015)

19. P.V. Malaji, S.F. Ali, Eur. Phys. J. Special Topics 224(14-15), 2823 (2015)

20. D. Tan, Y.G. Leng, Y.J. Gao, Eur. Phys. J. Special Topics 224(14-15), 2829 (2015)

21. F. Cottone, M. Mattarelli, H. Vocca, L. Gammaitoni, Eur. Phys. J. Special Topics 224(14-15), 2855 (2015)

22. D.X. Cao, S. Leadenham, A. Erturk, Eur. Phys. J. Special Topics 224(14-15), 2867 (2015)

23. Z. Hadas, C. Ondrusek, Eur. Phys. J. Special Topics 224(14-15), 2881 (2015)

24. S. Naifar, S. Bradai, C. Viehweger, O. Kanoun, Eur. Phys. J. Special Topics 224(14-15), 2897 (2015)

25. K. Kucab, G. Górski, J. Mizia, Eur. Phys. J. Special Topics 224(14-15), 2909 (2015)

26. S. Bradai, S. Naifar, C. Viehweger, O. Kanoun, G. Litak, Eur. Phys. J. Special Topics 224(14-15), 2919 (2015)

27. U. Javed, H.L. Dai, A. Abdelkefi, Eur. Phys. J. Special Topics 224(14-15), 2929 (2015)

28. J. Roscow, J. Taylor, C.R. Bowen, Eur. Phys. J. Special Topics 224(14-15), 2949 (2015)

29. A. Avent, C.R. Bowen, Eur. Phys. J. Special Topics 224(14-15), 2967 (2015)

30. S.F. Alazemi, A. Bibo, M.F. Daqaq, Eur. Phys. J. Special Topics 224(14-15), 2993 (2015)

31. L.L. Silva, S.A. Oliveira, P.M.C.L. Pacheco, M.A. Savi, Eur. Phys. J. Special Topics 224(14-15), 3005 (2015)

32. V. Piccirillo, A.M. Tusset, J.M. Balthazar, Eur. Phys. J. Special Topics 224(14-15), 3023 (2015)

33. F.C. Janzen, A.M. Tusset, V. Piccirillo, J.M. Balthazar, M. Silveira, R.M.L.R. da Fonseca Brasil, Eur. Phys. J. Special Topics 224(14-15), 3041 (2015) 